\title{
Hilar Gland Tuberculosis in Nepalese Adults Masquerading as Malignant Lymphoma
}

\author{
Capt J McCallum, RAMC \\ Major W Melia, MRCP, RAMC \\ Medical Division, British Military Hospital, Hong Kong \\ Major A Henderson, MRCP, RAMC \\ Physician \\ British Military Hospital Rinteln
}

SUMMARY: We report two Nepalese patients with tuberculosis, in whom the sole chest X-ray abnormality was hilar lymphadenopathy. This rare variant of tuberculosis, not previously described in Nepalese patients, may easily be mistaken for malignant lymphoma.

\section{Introduction}

Although the chest X-ray appearances of post-primary tuberculosis are extremely varied, the finding of hilar adenopathy as the sole abnormality appears to be rare in patients of European stock ${ }^{1}$. This variant of tuberculosis, which may easily be mistaken for malignant lymphoma, bronchial carcinoma or even sarcoidosis, has however been described in Indian and African adults ${ }^{1-7}$. We describe for the first time, this rare presentation in two Nepalese women and draw attention to the similarity of the clinical picture to malignant lymphoma.

\section{Case I}

A 26 year old Nepalese woman presented with a six week history of fever, malaise, weight loss of about $8 \mathrm{~kg}$ and a cough productive of white sputum. On examination a single enlarged right supraclavicular lymph node was palpable. Her Heaf test was strongly positive (Grade IV) and a chest X-ray showed right hilar lymphadenopathy but was otherwise normal (Fig 1). The ESR was $107 \mathrm{~mm} /$ hour, haemoglobin $10 \mathrm{~g} / \mathrm{dl}$, MCV $87 \mathrm{fl}$ and white cell count $4.7 \times 10^{9} / 1$ without any atypical cells. Examination of stool showed a hookworm infestation which was thought to account for the anaemia, but Ziehl-Neelsen stain of the sputum was negative for tubercle bacilli, as was culture on Lowenstein-Jensen medium. The clinical suspicion of tuberculosis was confirmed by histology of the excised supraclavicular lymphnode. Treatment with Rifinah 300 ii daily and ethambutol $15 \mathrm{mg} / \mathrm{kg} /$ day resulted in rapid clinical improvement and gradual resolution of the right hilar adenopathy (Fig 2). The ethambutol was discontinued after 8 weeks while the Rifinah will be continued for 9 months.

\section{Case II}

A 23 year old Nepalese woman presented with a 3 month history of non-productive cough, weight loss and a painful swelling in the left supraclavicular region. On examination she was pyrexial $\left(38^{\circ} \mathrm{C}\right)$ and had a single enlarged supraclavicular lymph node. A chest X-ray revealed right hilar lymphadenopathy but was otherwise normal. Heaf test was strongly positive (Grade IV), but tubercle bacilli were not present in the sputum. Her ESR was $117 \mathrm{~mm} /$ hour but haematological examination was otherwise normal. Biopsy of the supraclavicular lymph node showed tuberculosis. She was started on Rifinah 300 ii daily plus ethambutol $15 \mathrm{mg} / \mathrm{kg}$ with symptomatiơ and radiological improvement. The ethambutol wa. discontinued after 8 weeks. It is intended to continue th Rifinah 300 for 9 months.

\section{Discussion}

Radiological evidence of hilar lymphadenopathy rarely found in patients of European stock with pos primary tuberculosis.

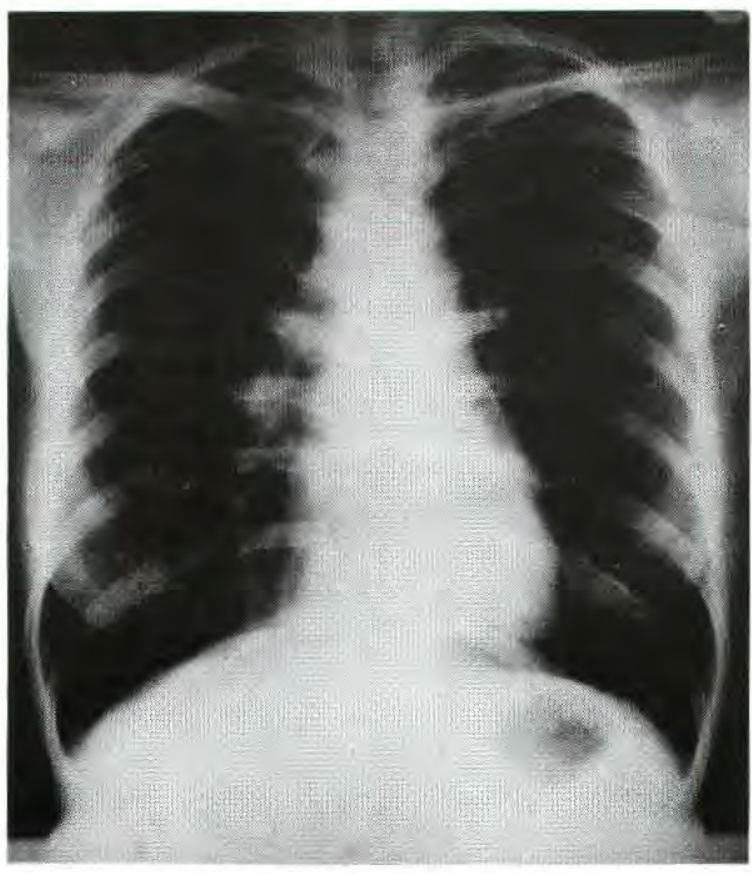

Fig 1. - Patient 1. Chest X-ray before treatment 


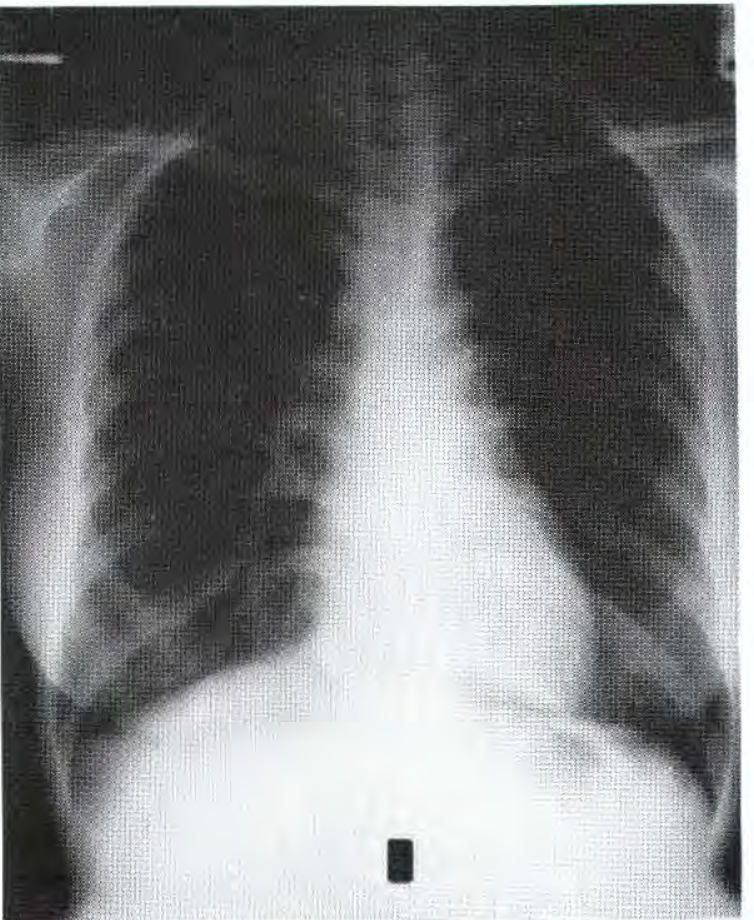

Fig 2. - Patient 1. Chest X-ray after 3 months of antituberculous chemotherapy

In the two patients described, the radiological features, coupled with fever, weight loss, supraclavicular lymphadenopathy and the enormously raised ESR were more suggestive of a malignant lymphoma. When superficial lymphadenopathy is present, as in our cases, a precise diagnosis can be rapidly arrived at by surgical biopsy. In patients without accessible lymph nodes however, more invasive procedures such as mediastinoscopy may be considered.

The diagnosis of tuberculosis might not immediately suggest itself in the absence of the more typical radiological features and of tubercle bacilli from the sputum. Although it has never been previously described in Nepalese, in Indians and Africans postprimary tuberculosis may rarely present with hilat lymphadenopathy as the sole chest $\mathrm{X}$-ray abnormality ${ }^{1-7}$.

The Heaf test then assumes a central diagnostic role. as a Grade IV reaction would favour tuberculosis, while being strongly against the diagnosis of lymphoma or sarcoidosis. We believe that the policy used by farrow et $\mathrm{al}^{7}$ for the management of Indians with hilar adenopathy can be safely extended to Nepalese patients. Those presenting with hilar adenopathy plus a strongly positive Heaf test should be treated for tuberculosis without resort to mediastinoscopy or biopsy. Invasive procedures should be reserved for those in whom the clinical picture is atypical, the blood film is abnormal or those who fail to respond to adequate antituberculosis chemotherapy.

\section{REFERENCES}

1. Croftov J and Douglas A. Respiratory Diseases. Third Edition. Blackwell Scientific Publications Oxford \& London. 1981.P. 272.

2. Silver C P and STFEL S J. Mediastinal lymphatic glan 끙 tuberculosis in Asian and coloured immigrants. Lance $\overparen{D}$ $1961 ; 1: 1254-1256$.

3. KENT D C and ElliotT R C. Hilar adenopathy in tuber culosis. Am Rev Respir Dis 1967; 96: 439.

A H. Mediastinescopy: a critical review of 220 cases. Ann Surg 1971; 174: 965-968.

5. Winterbauer R H. Belic $\mathrm{N}$ and MoOres $\mathrm{K}$. A clinici interpretation of bilateral hilar adenopathy. Ann Inter Med 1973; 78: 65-71.

6. BLoOMBERG T J and Dow C J. Contemporary mediastinal tuberculosis. Thorax 1980; 35: 392-396.

7. FARROW P R, et al. Thoracic lymphadenopathy in Asians resident in the United Kingdom: the role of mediastinoscopy in the initial diagnosis. Thorax 1985; 40: 121-124.

8. Crofton J and Perera H W. Tuberculosis Programme in Nepal. Assignment Report, November 1977-April 1978. WHODocument. Delhi, India.

9. Cumming G and Semple S J. Disorders of the Respiratory System. Blackwell Scientific Publications. Second edition 1980. P. 358. 